\title{
Iterated SISO MUD for Synchronous Uncoded CDMA Systems over AWGN Channel: Performance Evaluation in Overloaded Condition
}

\author{
Ferry P. Wathan, Reza Hoshyar and Rahim Tafazolli \\ Mobile Group, Centre for Communication Systems Research \\ University of Surrey, UK \\ Guildford GU2 7XH, Surrey, U.K. \\ E-mail: \{F.Wathan, R.Hoshyar, R.Tafazolli\}@surrey.ac.uk
}

\begin{abstract}
The performance of various iterated soft-input softoutput (SISO) multiuser detection (MUD) techniques is evaluated and compared for the case of overloaded condition for symbolsynchronous uncoded Code-Division Multiple Access (CDMA) systems over Additive White Gaussian Noise (AWGN) channel. We show that, via computer simulation, while the Bit-Error Rate (BER) performance of the serial version of the Symbol-Level iterated (SLi) SISO MUD technique is inferior to its parallel counterpart, its convergence rate is the faster between the two. Yet, the Chip-Level iterated (CLi) SISO MUD performs the best. Moreover, the Max-Log-MAP version of the CLi SISO MUD complementing the recently proposed Low-Density Signature (LDS) structure is introduced and compared to its Log-MAP version. The sensitivity of the various CLi SISO MUD techniques to the existence of the short-cycles in the underlying factor graph of the LDS structure is also analyzed. Our simulations confirm that the Max-Log-MAP version performs better compared to its more complex Log-MAP version in higher system loading condition. We also show that a performance close to single-user bound can be achieved at higher Signal-to-Noise Ratio (SNR) regime for system loading of up to $250 \%$.
\end{abstract}

\section{INTRODUCTION}

The quest for improving the system capacity is a never ending research subject in mobile wireless communication. In this paper, we focus on the maximization of the user capacity, defined as the maximum number of admissible users. Furthermore, the system of interest is the symbol-synchronous uncoded CDMA systems with equal-power and equal-rate users and the transmission is over the AWGN channel.

Let $N$ and $K$ be the processing gain and the number of active users, respectively. The soft-limit of user capacity in this kind of system is equal to $N$. However, when the bandwidth is at premium, e.g. the system described in [1], the need to go beyond that soft-limit is imminent. This condition is known as overloaded condition, defined when $K>N$.

Signal demodulation in overloaded condition for the system of interest is a major challenge as there exist rank-deficiency of the spreading signatures matrix of the system and, hence, orthogonalization of the signatures is impossible. From the literature, this problem can be tackled by utilizing an advanced signal processing in the receiver side and optimizing the spreading signatures in the transmitter side.
On the receiver side, MUD techniques can be used to demodulate the transmitted signal perturbed by noise. From the literature, optimum MUD techniques yield the best performance in terms of the achievable probability of error [2], yet their computational complexity is intractable for practical implementation. Therefore, the attention has been shifted towards sub-optimum MUD techniques. Moreover, the popular linear MUD techniques (see [3] for detailed treatment), e.g. linear Minimum Mean-Squared Error (MMSE), perform poorly in overloaded condition [4] due to its rank deficiency of the signature covariance matrix. However, recently, by feeding back the recently estimated symbols' soft values, SLi SISO MUD techniques that meet MMSE criteria have been shown to achieve a substantial improvement for uncoded CDMA systems [5].

Furthermore, on the transmitter side, signature optimization can also be used to improve the system performance. The optimum signature for overloaded condition is the signature that meets the Welch-Bound-Equality (WBE), devised in [6]. The design and performance of the complex-valued WBE sequences can be found in [7]. However, WBE-sequences suffer from scalability ${ }^{1}$ problems, because they are designed for specific $(N, K)$ systems. Another interesting signature optimization is the family of hierarchy of orthogonal sequences (HOS) that uses multiple orthogonal sequences to support overloaded condition [8].

Most recently, a Low-Density Signature (LDS) structure complemented with the sub-optimum CLi SISO MUD techniques has been proposed in [9]. The idea of LDS is to modify the interference pattern per chip by switching-off a large number of signature chips such that the optimum MUD can be approximated by using sub-optimum Message Passing Algorithm (MPA). Instead of optimizing the signature values, a different strategy in approximating the optimum MUD is taken by modifying the signature structure. By doing this, the scalability will not be a major problem in the system.

The contribution of this paper is twofold. First, we compare

${ }^{1}$ The scalability is one of the more important aspects in wireless communication in order to obtain a reliable and flexible system without having to compromise the signalling overhead each time a connection is terminated. 
the performance of the successive and parallel SLi SISO MUD techniques for CDMA systems with conventional as well as with the LDS structure. In addition, by utilizing LDS structure, CLi SISO MUD techniques are added for comparison. Second, we investigate the performance of the Max-Log-MAP version of the CLi SISO MUD with the LDS structure. The sensitivity of the various CLi SISO MUD techniques to the existence of short-cycles, which is known for having a bias effect for MPA, in the corresponding factor-graph of the LDS structure is also investigated. The Bit-Error Rate (BER) and the convergence behavior are be used as the performance metrics.

The remainder of this paper is organized as follows. Section II presents the system model used throughout this paper. Both the SLi and CLi SISO MUD techniques are introduced and explained in Section III. The simulation results are given in Section IV and Section V concludes this paper.

\section{SySTEM MODEL}

Consider a symbol-synchronous uncoded $(N, K)$ CDMA system for uplink transmission over AWGN channel. Let $x_{k}$ and $\mathbb{X}_{k}$ be, respectively, the transmitted symbol with period of $T_{s}$ and the constellation alphabet for user $k$. Without loss of generality and for notation brevity, all users are assumed to take their symbol from the same normalized constellation alphabet, i.e. $\mathbb{X}_{k}=\mathbb{X}, \forall k=1, \ldots, K$ with unit average energy. The received signal can be written as

$$
y(t)=\sum_{k=1}^{K} s_{k}(t) e^{j \theta_{k}(t)} A_{k} x_{k}(t)+\nu(t) ; 0 \leq t<T_{s}
$$

where $s_{k}(t), A_{k}$, and $\theta_{k}(t)$ represent, respectively, the spreading signature, the gain, and the user-specific phase ${ }^{2}$ for user $k=1, \ldots, K$ at time instant $t$. The thermal noise at the receiver is represented by $\nu(t) \sim \mathcal{C N}\left(0, \sigma^{2}\right)$. Assuming a perfect synchronization, we can rewrite (1) in its discrete form as follows.

$$
\begin{aligned}
\mathbf{y} & =\sum_{k=1}^{K} \mathbf{h}_{k} x_{k}+\mathbf{v} \\
& =\mathbf{H} \mathbf{x}+\mathbf{v}
\end{aligned}
$$

where $\mathbf{y}=\left[y_{0}, \ldots, y_{N-1}\right]^{T}, \mathbf{h}_{k}=\left[h_{k, 0}, \ldots, h_{k, N-1}\right]^{T}$ and $\mathbf{v}=\left[v_{0}, \ldots, v_{N-1}\right]^{T}$ are the received signal, the effective signature for user $k$ and the noise vectors of size $N$, respectively, which are formed by stacking $N$ successive chips together. Furthermore, matrix $\mathbf{H}=\left[\mathbf{h}_{1} \ldots \mathbf{h}_{K}\right]$ represents the effective signature of all users in the system, where the $n$ th component of the effective signature for user $k$ is given by $h_{k, n}=A_{k} e^{j \theta_{k}(t)} s_{k, n}$. The most recently proposed LDS structure works by switching-off a large number of spreading chips. Let $d_{v} \ll N$ be the maximum number of non-zero elements in user's signature or the effective processing gain. After spreading, a user-unique interleaver is used to randomize

\footnotetext{
${ }^{2}$ The phase is assumed randomly distributed. This phase information is common for practical system implementation and, for this paper, is important for system analysis.
}

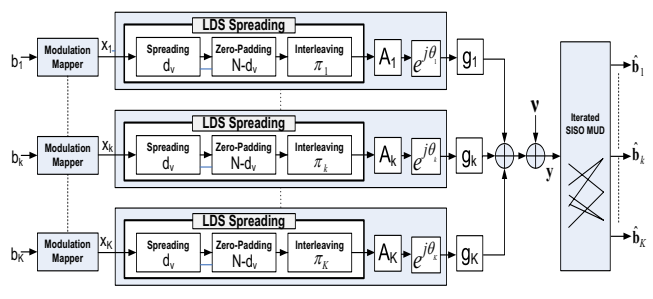

Fig. 1. LDS System Model

the signal. By employing LDS structure, the system model is depicted in Figure 1.

Denote $\mathbf{w}_{k}$ and $\mathbf{W}=\left[\mathbf{w}_{1}, \ldots, \mathbf{w}_{K}\right]$ as the indicator vector, after interleaving, for user $k$, which is the all zeros vector but contains ones in $d_{v}$ positions and the system's indicator matrix. The interleaving is designed such that the matrix $\mathbf{W}$ is lowdensity, as in the parity check matrix of LDPC. Consequently, the number of interfering users per chip is now $d_{c} \ll K$. Let $\xi_{n}$ and $\zeta_{k}$ be the set of users participating in chip $n$ and the set of chips, over which user $k$ spreads their symbol, respectively. Then, with LDS structure, the received signal sampled at chip $n$ is given by

$$
\begin{aligned}
y_{n} & =\sum_{k \in \xi_{n}} h_{k, n} x_{k}+\nu_{n} \\
& =\mathbf{h}_{[n]}^{T} \mathbf{x}^{[n]}+\nu_{n}
\end{aligned}
$$

where $\mathbf{h}_{[n]}$ and $\mathbf{x}^{[n]}$ denote a vector comprising effective signature components and the symbols of all interfering users in $n$-th chip.

\section{ITERATED SISO MUD TECHNIQUES}

\section{A. Symbol-Level Detection}

The SLi SISO MUD can be implemented either successively or in parallel. To the best of our knowledge, the performance comparison for these two implementations has not been yet available in the literature, let alone for the overloaded condition. In this paper, we use the same approach as in [10] to construct the MMSE-based SLi SISO MUD and, for completeness, it is described as follows. The prior probabilities, which are computed either from initial assumption or from the previous iteration stage, are used to compute the softestimates (i.e. the mean value) of the user $k$ symbol, denoted as $\tilde{x}_{k}=\sum_{x_{k} \in \mathbb{X}} x_{k} P\left(x_{k}\right)$. Then, the following vectors can be formed.

$$
\begin{aligned}
\tilde{\mathbf{x}} & =\left[\tilde{x}_{1} \ldots \tilde{x}_{K}\right]^{T} \\
\tilde{\mathbf{x}}_{\mathbf{k}} & =\tilde{\mathbf{x}}-\tilde{x}_{k} \mathbf{e}_{k}
\end{aligned}
$$

where $\mathbf{e}_{k}$ denotes the all-zero vector but its $k$-th component has value of one. In order to remove the interference using the soft-estimates above, soft interference cancellation (IC) is made such that

$$
\tilde{\mathbf{y}}_{k}=\mathbf{y}-\mathbf{H} \tilde{\mathbf{x}}_{\mathbf{k}}
$$


where $\tilde{\mathbf{y}}_{k}$ represents the vector of received signal with some effect of the interferers are removed. To further remove the residual interference that might still exist, linear filter, $\tilde{\mathbf{f}}_{k}$, is used such that $\mathrm{E}\left[\left\|x_{k}-\tilde{\mathbf{f}}_{k}^{H} \tilde{\mathbf{y}}_{k}\right\|^{2}\right]$ can be minimized.

$$
z_{k}=\tilde{\mathbf{f}}_{k}^{H} \tilde{\mathbf{y}}_{k} \text {. }
$$

Assuming that the residual noise and interference from linear MMSE detector can be approximated with a Gaussian distribution $^{3}$, the estimated symbol $z_{k}$ can be written as

$$
z_{k}=\mu_{k} x_{k}+\eta_{k} ; \quad \eta_{k} \sim \mathcal{C N}\left(0, v_{k}^{2}\right) .
$$

From (9), the symbol probabilities of $x_{k}$ can be calculated as

$$
P\left(\hat{x}_{k}\right) \propto \exp \left(-\frac{1}{2 v_{k}^{2}}\left\|z_{k}-\mu_{k} x_{k}\right\|^{2}\right) .
$$

For parallel MMSE-based SLi SISO MUD technique, at $j$ th iteration, the estimated posterior probability $P\left(\hat{x}_{k}\right)$ will not be used to calculate (7)-(10) for user $k+1$. However, if serial MMSE-based SLi SISO MUD is used, the probabilities of user $k$ will be updated with the newly estimated values and will be used for estimating the next user $k+1$. Note that the domino effect normally seen in successive processing will not be as detrimental as when the hard-values are used for serial processing. However, to have a fair comparison, the users are sorted prior the computation by using the user ordering algorithm proposed in [12]. The drawback with MMSE-based SLi SISO MUD techniques, either with parallel or serial structure, is that the linear MMSE filter $\tilde{\mathbf{f}}_{k}$, which includes an inversion matrix of size $K \times K$, needs to be updated for every user, every iteration and every symbol. In AWGN channel, this computation, assuming that the inverse of signature covariance matrix is a priori provided, can be done recursively via Matrix Inversion Lemma as highlighted in [10]. Nevertheless, for a large $K$, this computation could be expensive.

\section{B. Chip-Level Detection}

Graphical models such as Factor Graph (interested reader can refer to the excellent review about factor graph in [13] and references therein) allow an easy implementation and analysis of many iterative processing techniques, e.g. iterative joint detection/decoding techniques have been derived from an appropriate factor graph in [14], [15].

Denote $\mathcal{G}(\mathcal{U}, \mathcal{C})$ as the underlying factor graph of un-coded $(N, K)$ CDMA systems, where the users $(\mathrm{k}=1, \ldots, \mathrm{K})$ and the chips $(\mathrm{n}=1, \ldots, \mathrm{N})$ are represented by variable nodes $u \in \mathcal{U}$ and function nodes $c \in \mathcal{C}$, respectively. An edge $e(k, n)$ exists if the received chip $y_{n}$ contains the symbol from user $k$ and is shown as a line connecting variable node $u_{k}$ and function node $c_{n}$.

Following the aforementioned description, the factor graph of the conventional CDMA structure is illustrated as a forestlike, where each variable node is connected to all function

\footnotetext{
${ }^{3}$ We acknowledge, however, that the noise and residual MAI can be well approximated by using Gaussian Distribution in the first iteration [11]. Therefore this assumption is biased when used for iterated SISO MUD.
}

nodes. This fact makes the implementation of MPA on the factor-graph inefficient for uncoded CDMA systems.

Most recently, an LDS structure has been proposed in [9] and, due to the sparse nature of its factor graph, LDS structure allows an effective implementation of the MPA. Consequently, a new CLi SISO MUD can be designed. However, it is worth mentioning that, even though MAP can approximate the optimum MAP detection, the posterior probability of the MPA-based CLi SISO MUD, due to the existence of short cycles, can only approach its exact value.

The generic form of CLi SISO MUD can explained as follow. Let $\mathcal{L}_{n \downarrow}^{j}\left(x_{k}\right)$ and $\mathcal{L}_{n \uparrow}^{j}\left(x_{k}\right)$ be the messages ${ }^{4}$ sent along edge $e_{n, k}$, at $j$-th iteration, from variable node $u_{k}$ to function node $c_{n}$ and vice-versa, respectively. Assuming there is no a priori probabilities available, the initial messages $(j=0)$ are set to zeros: $\mathcal{L}_{n \downarrow}^{0}\left(x_{k}\right)=0, \forall k, \forall n$. The messages are updated using the following rules:

$$
\begin{aligned}
\mathcal{L}_{n \downarrow}^{j}\left(x_{k}\right) & =\sum_{m \in \xi_{k} \backslash n} \mathcal{L}_{m \uparrow}^{j-1}\left(x_{k}\right) \\
\mathcal{L}_{n \uparrow}^{j}\left(x_{k}\right) & =\mathcal{F}\left(x_{k} \mid y_{n}, \mathcal{L}_{n \downarrow}^{j}\left(x_{l}\right), \forall l \in \zeta_{k} \backslash k\right)
\end{aligned}
$$

It can be easily seen from (11) and (12) that all messages are restricted to consist of only the extrinsic information [13].

In order to approximate the optimum MAP (or Log-MAP as the messages are represented in their logarithmic-values) detector, as derived in [9], the function in (12) must calculate the local marginalization and can be written as

$$
\mathcal{F}(\bullet)=\log \left(\sum_{\substack{\mathbf{x}[n] \in \mathbb{X}^{d_{c}} \\ x_{k}}} p^{j}\left(y_{n} \mid \mathbf{x}^{[n]}\right) \prod_{l \in \xi_{n} \backslash k} P_{n}^{j}\left(x_{l}\right)\right)
$$

where

$$
\begin{aligned}
p^{j}\left(y_{n} \mid \mathbf{x}^{[n]}\right) & \propto \exp \left(-\frac{1}{\left(2 \sigma^{2}\right)}\left\|y_{n}-\mathbf{h}_{[n]}^{T} \mathbf{x}^{[n]}\right\|^{2}\right) \\
P_{n}^{j}\left(x_{l}\right) & =\exp \left(\mathcal{L}_{n \downarrow}^{j}\left(x_{l}\right)\right) .
\end{aligned}
$$

Combining (14) and (15) into (13), the message update is given by

$$
\mathcal{L}_{n \uparrow}^{j}\left(x_{k}\right)=\kappa_{n, k} \max _{\substack{\mathbf{x}^{[n]} \in \mathbb{X}^{d_{c}} \\ x_{k}}}\left(\sum_{l \in \xi_{n} \backslash k} \mathcal{L}_{n \downarrow}^{j}\left(x_{l}\right)-\frac{1}{2 \sigma^{2}}\left\|y_{n}-\mathbf{h}_{[n]}^{T} \mathbf{x}^{[n]}\right\|^{2}\right)
$$

where $\kappa_{n, k}$ denotes the normalization ${ }^{5}$ coefficient and

$$
\begin{aligned}
\max ^{\star} & =\log \left(e^{a}+e^{b}\right) \\
& =\max (a, b)+\underbrace{\ln \left(1+\exp \left(-|a-b|^{2}\right)\right)}_{\varsigma}
\end{aligned}
$$

is the numerically stable function derived by using Jacobian algorithm in [17], where $\varsigma$ is known as the correction factor. This detector is termed CLi SISO Log-MAP MUD. This

${ }^{4}$ Message is defined as a vector comprising the reliability values of the adjacent variable node. Throughout this paper, the message is represented by its computationally efficient and stable Logarithmic-value.

${ }^{5}$ It has been suggested in [16] that the normalization can provide the added stability of the algorithm and avoid numerical underflow. 
algorithm will output the exact posteriori probability when the underlying factor graph is cycle-free. However, as we want to have finite number of iterations, we relax this definition such that the factor graph is called cycle-free when its girth, defined the minimum cycle of the variable node in the system, is bigger than six.

Furthermore, it is easy to see that, by utilizing local marginalization, the complexity of the receiver has been reduced so that it is exponential only to $d_{c}$ instead of to $K$, where $d_{c} \ll k$. In relevant literature, having assigned $\mathcal{F}(\bullet)$ according to (16), this MPA technique is also known as Sum-Product Algorithm (SPA) [13] or, following Pearl's terminology of belief propagation [16], as Belief Update. In [9], it has been shown that, by using CLi Log-MAP detector, the system with $200 \%$ loading condition can achieve a performance very close to single-user bound (SUB). The local marginalization shown in (16) utilizes the $\max ^{\star}$ operator. To reduce the complexity, max operator can be assigned instead and, hence, the message update (12) is given by

$\mathcal{L}_{n \uparrow}^{j}\left(x_{k}\right)==\kappa_{n, k} \max _{\substack{\mathbf{x}^{[n]} \in \mathbb{X}^{d_{c}} \\ x_{k}}}\left(\sum_{l \in \xi_{n} \backslash k} \mathcal{L}_{n \downarrow}^{j}\left(x_{l}\right)-\frac{1}{2 \sigma^{2}}\left\|y_{n}-\mathbf{h}_{[n]}^{T} \mathbf{x}^{[n]}\right\|^{2}\right)$.

By replacing the $\max ^{\star}$ with the max operator, the resultant technique is now termed CLi SISO Max-Log-MAP MUD. This new technique will not give a correct marginalization even when the underlying factor graph is cycle-free. Nevertheless, it can lead to correct assignments.

\section{Simulation Results}

In this section, we present the simulation results for various SLi and CLi SISO MUD techniques presented in previous sections. Throughout this paper, Binary Phase Shift Keying (BPSK) is used to modulate the information symbols, whereas the transmission is done over AWGN channel. In addition, all users in the system have equal-rate and equal-power.

\section{A. Performance of Various SLi SISO MUD Techniques}

We compare the performance of various SLi SISO MUD techniques that meet MMSE criteria for overloaded synchronous uncoded CDMA systems with $N=12$ and $K=$ 16 with various signature structures, namely conventional and LDS structures. The signature for conventional structure is optimized using HOS approach; The HOS signature is designed by creating two sets of orthogonal signatures, by using Gram-Schmidt orthogonalizing algorithm. Each set can accommodate 12 users and 4 users, respectively. In addition, the (hard) MMSE-PPIC with the optimized smoothing coefficients is included for comparison. The random signatures with conventional structure is also included in order to evaluate the improvement made by utilizing HOS-optimized signatures.

Figure 2 depicts that the parallel SLi SISO MUD outperforms its serial version in any SNR moderate-to-high SNR regimes by using any structures. Furthermore, as expected, the performance of SLi MUD that uses hard-values is inferior to the SLi SISO MUD that utilizes soft-values. Interestingly,

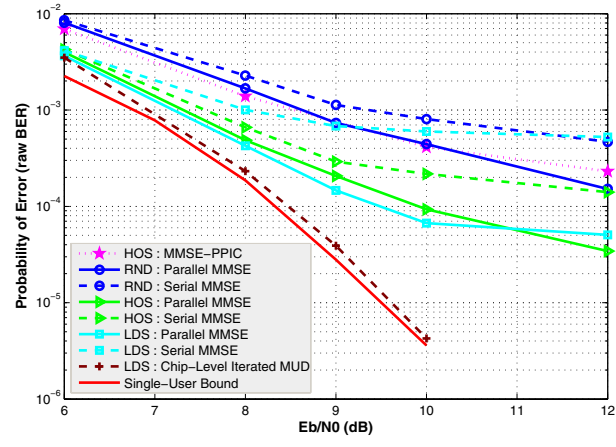

Fig. 2. BER Performance of Various SLi SISO MUD Techniques

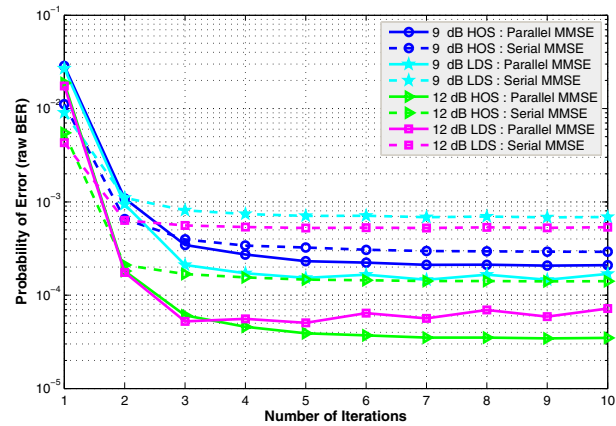

Fig. 3. Convergence Behavior of Various SLi SISO MUD Techniques

the performance of the parallel SLi SISO MUD with LDS structure is superior to that with HOS-optimized conventional structure at $E_{b} / N_{0} \leq 11 \mathrm{~dB}$. In addition, the CLi SISO MUD is shown the outperform any SLi SISO MUD techniques.

Figure 3 shows that the serial SLi SISO MUD gives the best first estimate and also converges the fastest, but not necessarily the best, in moderate-to-high SNR. It is to be noted that by using LDS structure, the paralel SLi SISO MUD experiences more oscillatory behavior in the higher SNR regimes. However, this behavior cannot be observed when the optimized conventional signature structure is used.

In thesis paper, we intentionally do not include the fading channel into our performance comparison as we concentrates on AWGN channel, where the interference is mainly caused by the non-orthogonality of the signatures in overloaded condition. However, from literature (cf. [18]), there exist some power-control mechanism that can well assist the signal demodulation in overloaded condition. Assuming the fading channel can create more degree of freedom in signal demodulation, e.g. through user-ordering mechanism at the receiver, some performance gain can be achieved and may lead to different conclusion when comparing the performance the successive and parallel structure of the MMSE-based SLi SISO MUD techniques. 


\section{B. User Capacity}

In this sub-section, the performance of LDS structure for synchronous overloaded CDMA-based systems over AWGN channel is investigated via computer simulation. Various LDS structures are presented with different loading conditions. The LDS structure whose variable nodes have the same density of $d_{v}$ and whose function nodes have the same density of $d_{c}$ is called regular LDS. Furthermore, the performance of various CLi SISO MUD when the user nodes of the underlying factor graph have short cycles, defined as Cycle-of-Four $(\mathrm{CoF})$, are investigated. In order to summarize the performance of $\mathrm{CLi}$ SISO MUD, several LDS structures, denoted as LDS $N \times K$, are introduced and can be found in Table I.

TABLE I

General Detail of Various LDS Structures

\begin{tabular}{|c||c|c|c|c|}
\hline LDS Schemes & $\beta$ & Regularity & $d_{v}$ & $\% \mathbf{C o F}$ \\
\hline $60 \times 80$ & 1.333 & $\checkmark$ & 3 & 10 \\
\hline $60 \times 120 a$ & 2.000 & $\checkmark$ & 3 & 31 \\
\hline $60 \times 120 b$ & 2.000 & $\checkmark$ & 3 & 0 \\
\hline $100 \times 250$ & 2.500 & & 3 & 0 \\
\hline
\end{tabular}

First, we present the system performance of the two MUD techniques in various LDS structures. From Figure 4, we can see that for slightly overloaded condition, i.e. $\beta=1.33$, the Max-Log-MAP version of the CLi SISO MUD have almost similar performance to its Log-MAP version. However, as we increase the system loading, by designing an appropriate LDS matrix, the Max-Log-MAP version outperforms its LogMAP counterpart, especially at the LDS $100 \times 250$ where the loading is $250 \%$. Figure 5 depicts the convergence behavior of both MUD techniques. It suggests that, in AWGN channel, the performance of the Max-Log-MAP version almost always converges, while the Log-MAP version, at higher system loading, tends to oscillate and, therefore, cannot find the fixed points.

\section{Effect of Short-Cycles}

Furthermore, Figure 6 shows that the existence of multiple $\mathrm{CoF}$ of upto $31 \%$ in the LDS structure with the same loading condition causes the system performance to degrade slightly. Furthermore, the Max-Log-MAP version of the CLi SISO MUD tends to be superior to its Log-MAP counterpart. This phenomenon can be understood intuitively. When the factor graph has multiple $\mathrm{CoF}$, the incoming messages will contain the previously outgoing messages. We argue that this information is mistakenly accepted as a new information and the message update in (16) double-counts this information, due to the correction factor given in (17). However, when (18) is utilized to revise the message, double-count will not exist. However, it is important to highlight that while the Max-LogMAP version do not give the correct posterior probability, it may still lead to a correct assignment.

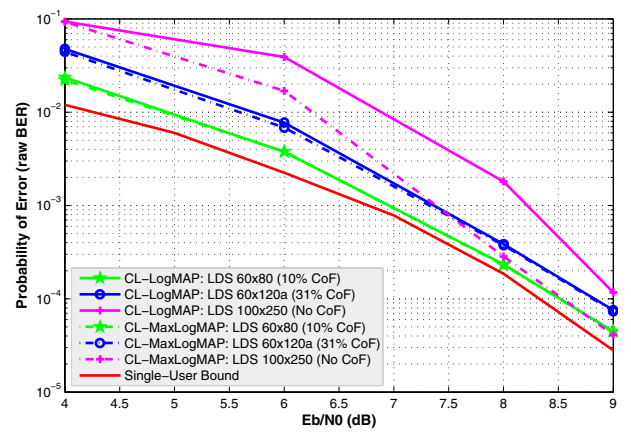

Fig. 4. Performance of Various CLi SISO MUD Techniques

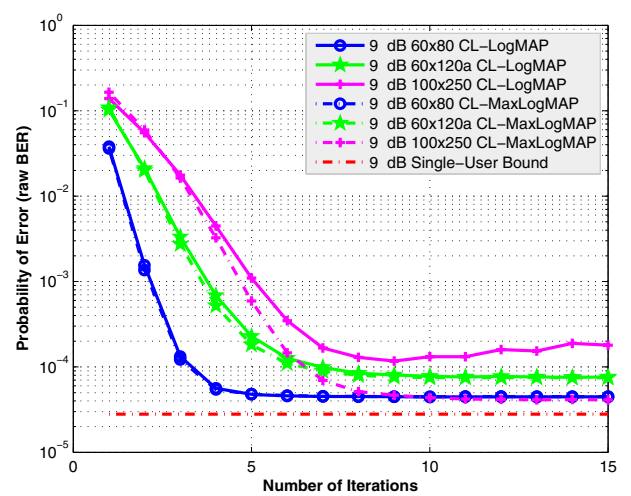

Fig. 5. Convergence Behavior of various CLi SISO MUD Techniques

\section{CONCLuSions}

In this paper, we compare the performance of the successive and parallel SLi SISO MUD techniques, which meet MMSE criteria, complemented with HOS-optimized spreading signatures for symbol-synchronous uncoded CDMA systems in overloaded condition. We show that, while the serial version almost always converges and it converges faster, its parallel counterpart performs the better. Furthermore, we present and analyze the Max-Log-MAP version of CLi SISO MUD, which is the extension of previously proposed CLi SISO Log-MAP MUD, with CDMA systems utilizing LDS structure. Our simulation confirms that the CLi SISO MUD complemented with LDS structure outperform any SLi SISO MUD techniques utilizing conventional structure.

Interestingly, in higher system loading, the Max-Log-MAP version of the CLi SISO MUD outperforms the more complex Log-MAP counterpart, while in lower system loading, their performance is almost identical. Moreover, our simulations also suggest that the Max-Log-MAP version is less sensitive to the existence of multiple $\mathrm{CoF}$ because it avoids the double counting of the messages.

The analysis on, practically, more realistic coded-CDMA scenario will be addressed in our future work. 


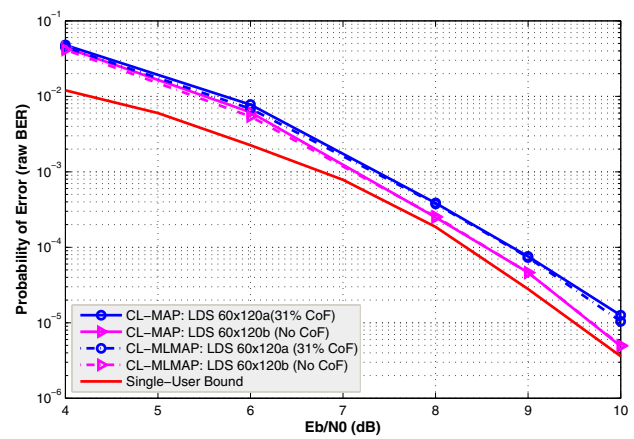

Fig. 6. The Effect of the Existence of Multiple $\mathrm{CoF}$

\section{REFERENCES}

[1] M. Varanasi and T. Guess, "Bandwidth-efficient multiple access (BEMA): A new strategy based on signal design for multiuser receivers under quality-of-service constraints," IEEE Trans. Commun., vol. 49, no. 5, pp. 844-854, May 2001.

[2] S. Verdú, "Minimum probability of error asynchronous gaussian multiple-access channels," IEEE Trans. Inform. Theory, vol. 32, pp. 8594, Jan. 1986

[3] — Multiuser Detection. New York: Cambridge Univ. Press, 1998.

[4] M. Varanasi, C. Mullis, and A. Kapur, "On the limitation of linear mmse detection," IEEE Trans. Inform. Theory, vol. 52, no. 9, pp. 4282-4286, Sept. 2006.

[5] A. Lampe et al., "A novel iterative multiuser detector for complex modulation schemes," IEEE J. Select. Areas Commun., vol. 20, no. 2, pp. 339-350, Feb. 2002.

[6] L. Welch, "Lower bounds on the maximum cross-correlation of signals," IEEE Trans. Inform. Theory, vol. 20, pp. 397-399, 1974.

[7] P. Cotae, "Transmitter adaptation algorithm for multicelullar synchronous DS-CDMA systems with multipath," IEEE J. Select. Areas Commun., vol. 24, no. 1, pp. 94-103, Jan. 2006.

[8] F. Vanhaverbeke and M. Moeneclaey, "Sum capacity of equal-power users in overloaded channels," IEEE Trans. Commun., vol. 53, no. 2, pp. 228-233, Feb. 2005.

[9] R. Hoshyar, F. Wathan, and R. Tafazolli, "Novel low-density signature structure for synchronous DS-CDMA systems," in Proc. IEEE Globecom'06, San Francisco, USA, Nov. 2006, pp. 1-5.

[10] X. Wang and H. Poor, "Iterative (turbo) soft interference cancellation and decoding for decoded CDMA," IEEE Trans. Commun., vol. 77, no. 7, pp. 1047-1061, July 1999.

[11] V. Poor and S. Verdu, "Probability of error in MMSE multiuser detection,” IEEE Trans. Inform. Theory, vol. 43, no. 3, pp. 858-871, May 1997.

[12] M. Varanasi, "Decision feedback multiuser detection: A systematic approach," IEEE Trans. Inform. Theory, vol. 45, no. 1, pp. 219-240, Jan. 1999.

[13] F. Kschischang, B. Frey, and H. Loeliger, "Factor graphs and the sumproduct algorithm," IEEE Trans. Inform. Theory, vol. 47, no. 2, pp. 498-519, Feb. 2001

[14] J. Boutros and G. Caire, "Iterative multiuser joint decoding: Unified framework and asymptotic analysis," IEEE Trans. Inform. Theory, vol. 48, no. 7, pp. 1772-1793, July 2002.

[15] R. Drost and A. Singer, "Factor-graph algorithms for equalization," IEEE Trans. Signal Processing, vol. 55, no. 5, pp. 2052-2065, May 2007.

[16] J. Pearl, Probabilistic Reasoning in Intelligent Systems: Networks of plausible inference, 2nd ed. San Mateo, CA:Morgan Kaufmann, 1988.

[17] A. Viterbi, "An intuitive justification and a simplified implementation of the MAP decoder for convolutional codes," IEEE J. Select. Areas Commun., vol. 16, no. 2, pp. 260-264, Feb. 1998.

[18] G. Romano, F. Palmieri, and P. Willett, "Soft iterative decoding for overloaded CDMA," in Proc. Int. Conf. on Acoust.Speech, Signal Proc. ICASSP'05, vol. 3, Philadelphia, PA, USA, Mar. 2005, pp. 733-736. 